Peter Bachmann

\title{
Chaos in 1d Radiative Edge Plasmas
}

IPP 8/17

September 2001 


\section{MAX - PLANCK - INSTITUT FÜR PLASMAPHYSIK \\ GARCHING BEI MÜNCHEN}

Chaos in 1d Radiative Edge Plasmas

Peter Bachmann

IPP 8/17

September 2001

Max-Planck-Institut für Plasmaphysik, EURATOM Association Bereich Plasmadiagnostik, Mohrenstr. 41, 10117 Berlin ved." 


\title{
Chaos in 1d Radiative Edge Plasmas P. BACHMANN
}

\author{
Max-Planck-Institut für Plasmaphysik, \\ Bereich Plasmadiagnostik, EURATOM Association, \\ Mohrenstr. 41, D-10117 Berlin, Germany \\ e-mail: bachmann@ipp.mpg.de
}

\begin{abstract}
Bifurcation and chaos in radiative edge plasmas are investigated on the basis of a periodically driven reaction-diffusion equation which results from the time dependent $1 \mathrm{~d}$ heat conduction equation including a given periodically time-modulated impurity density. The temporal problem shows the transition to chaos through the Feigenbaum route. In $1 d$ and time dependent plasmas Hopf bifurcation and intermittency phenomena are shown to exist. The application to a carbon seeded plasma shows the existence of different oscillation regimes.
\end{abstract}




\section{Contents}

1 Introduction $\quad 3$

2 Periodically driven reaction-diffusion equation 3

3 Time dependent problem: Feigenbaum route 4

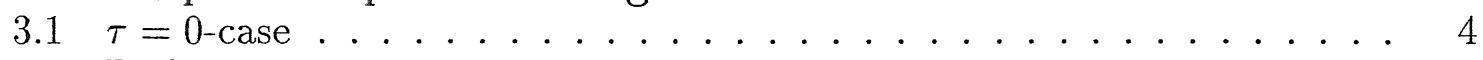

3.2 Radiative map . . . . . . . . . . . . . . . . 5

3.3 Attractors, period doubling and chaos . . . . . . . . . . 6

4 Steady problem $\quad 10$

5 Spatio-temporal chaos $\quad 12$

6 Application to Carbon $\quad 15$

7 Summary 16 


\section{Introduction}

Temperature bifurcations in radiative edge plasmas are relevant mechanisms that affect stability. They are caused by the non-monotonic dependence of the radiation function on the temperature, and the non-linear relation between the heat flux to the target plates and the temperature in high-recycling regimes [1] - [7].

In the frame of a bifurcation treatment low-frequency oscillations observed in divertor plasmas of the ASDEX Upgrade tokamak [8] have been interpreted in [9] by the use of [10]. These relaxation oscillations appear before marfe formation is initiated by successively enhancing the plasma density where the marfe acts as a precursor of the density limit caused by a radiative instability [11]. The simple 0-dimensional model used in [9] considers impurity production by sputtering and the temperature balance equation including heating and radiation processes. A variety of different oscillation regimes for densities and temperature has been found. The measured "small" oscillations can satisfactorily be explained by this model [12].

In order to investigate radiative properties of edge plasmas we make use of the model [9] and simplify furthermore by suppressing the equation for the density and assuming a given relaxation-like temporal modulation of the impurity density. This gives us the opportunity to arrange relaxation phenomena in a wider frame of temperature bifurcations which may also lead to temperature chaos. We describe the density modulation in the simple form of a periodic delta function behaviour that describes, strictly speaking, periodically injected impurities. It is the goal of this paper to show that this mechanism force temperature bifurcation and chaos in radiative edge plasmas.

Different to [9] heat conduction is taken into consideration. The model equation used is the $1 \mathrm{~d}$ and time dependent heat conduction equation which leads with the given ansatz to a periodically driven reaction-diffusion equation [13] (sect. 2). At first the temporal problem (sect. 3) will be considered which shows the transition to chaos through the Feigenbaum route. In sect. 4 a short insight is given into steady boundary value problems to be known (cp. appendix of [7]) for solving the spatio-temporal task that will be treated in sect. 5 .

\section{Periodically driven reaction-diffusion equation}

The starting point is the $1 \mathrm{~d}$ thermal conduction equation for the temperature $u(x, t)$ using dimensionless variables (cp. [7]):

$$
\begin{gathered}
\frac{\partial u}{\partial t}=\frac{\partial}{\partial x} \kappa(u) \frac{\partial u}{\partial x}+ \\
f(u, x, t), x \in\left[x_{0}, x_{n}\right], t \in\left[t_{0}, t_{n}\right] \\
\kappa(u) \equiv \kappa_{0} u^{\delta}
\end{gathered}
$$

$\kappa$ - heat conduction coefficient

$f$ is the source function given by

$$
f(u, x, t) \equiv H(x)-Q(u, x, t)-\beta u
$$


$\beta$ - damping coefficient, $H$ is the heating function,

$$
H(x) \equiv f_{s} s(x) \equiv f_{s} \exp \left[-\frac{\left(x-x^{*}\right)^{2}}{\Delta_{s}}\right]
$$

$f_{s}$ its strength. $Q$ is the loss function (with the strength $f_{q}$ ) assumed to be given by

$$
Q(u, x, t) \equiv f_{q} q(u, x, t) \equiv f_{q} \Theta_{\tau}(t) \sum_{m=1}^{M} c_{m}(x) \exp \left[-\frac{\left(u-u_{m}^{*}\right)^{2}}{\Delta_{q m}}\right]
$$

$Q$ is proportional to the impurity density $n_{z}$ which is assumed to be time modulated by the $\Theta_{\tau}(t)$ function where $\tau$ is the time period. We apply here the simple case of $\delta$ function modulation which allows an analytical treatment of the temporal problem, sect. 3 ,

$$
\Theta_{\tau}(t) \equiv \tau \sum_{i=1}^{\infty} \delta(t-i \tau), \Theta_{\tau \rightarrow 0}=1
$$

Strictly speaking, (6) describes the impurity injection with $\tau$ being the time period between two subsequent injections. The limiting $\tau \rightarrow 0$ represents the well-known case of time independent radiation $(\tau=0$-case). With the ansatz (6) eq. (1) represents a periodically driven reaction-diffusion equation [13].

\section{Time dependent problem: Feigenbaum route}

Now we are considering the time dependent problem (neglecting heat conduction) with $s(x)=1, c_{i}=$ const:

$$
\frac{d u}{d t}=f(u, t)
$$

\section{1 $\tau=0$-case}

Applying the explicit Euler scheme to eq. (7) for $\Theta_{\tau}=1$ leads with

$$
t_{i} \equiv t_{0}+(i-1) \Delta t, \Delta t \equiv \frac{t_{n}-t_{0}}{n-1}, i=1 \ldots n
$$

to the iterated map

$$
u_{i+1}=u_{i}+\Delta t f\left(u_{i}\right), \Theta_{\tau}=1, \Delta t \rightarrow 0
$$

The rest points of eq. (7) which are identical with the fix points of (9) for $\tau=0$ can be calculated analytically for $\beta=0, c_{1}=1$ and $M=1^{1}$ with the result:

$$
u_{1,2}^{0}=u_{1}^{*} \pm \sqrt{\Delta_{q 1} \ln \frac{f_{q}}{f_{s}}}, f_{s}<f_{q}
$$

Their stability follows from a linear perturbation analysis:

\footnotetext{
${ }^{1}$ The results can easily be generalized for $M>1$.
} 


$$
\left[\frac{d}{d t}-f^{\prime}\left(u^{0}\right)\right] \delta u=0, \delta u \equiv u-u^{0} \propto e^{L t}
$$

resulting in the eigen values (Lyapunov exponents)

$$
L_{1,2}=-f_{q} q^{\prime}\left(u_{1,2}^{0}\right)= \pm \frac{2 f_{s}}{\Delta_{q 1}} \sqrt{\Delta_{q 1} \ln \frac{f_{q}}{f_{s}}}
$$

There are two singular points $u_{1,2}^{0}$ for $f_{s}<f_{q}$ of which first one is unstable and the other one stable, cp. Fig. 1.

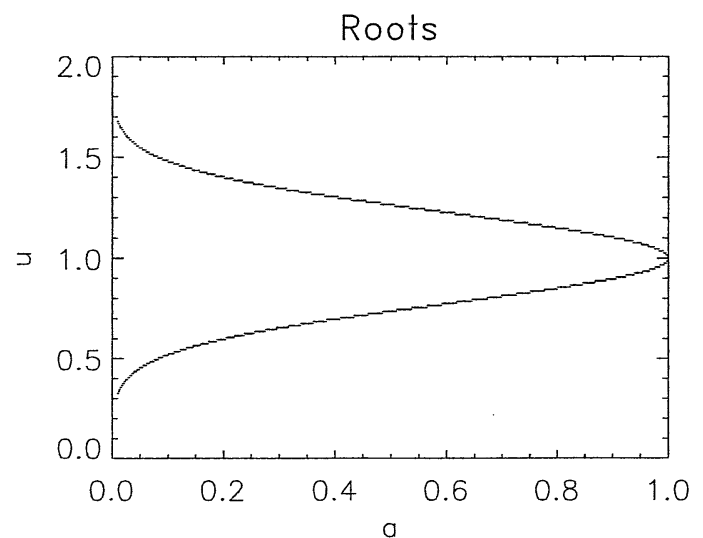

Attractors

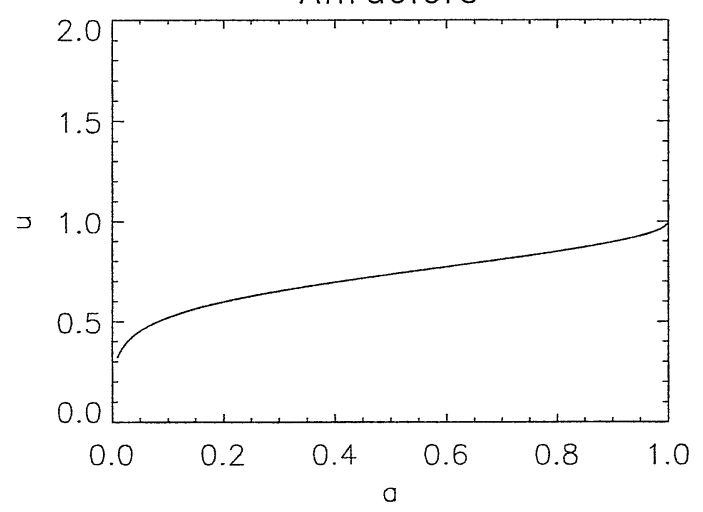

Fig. 1: Roots eq. (11) and attractors of the radiative map eq. (13) for $\tau=0.1$ and parameters $\mathrm{P} 1$ (15).
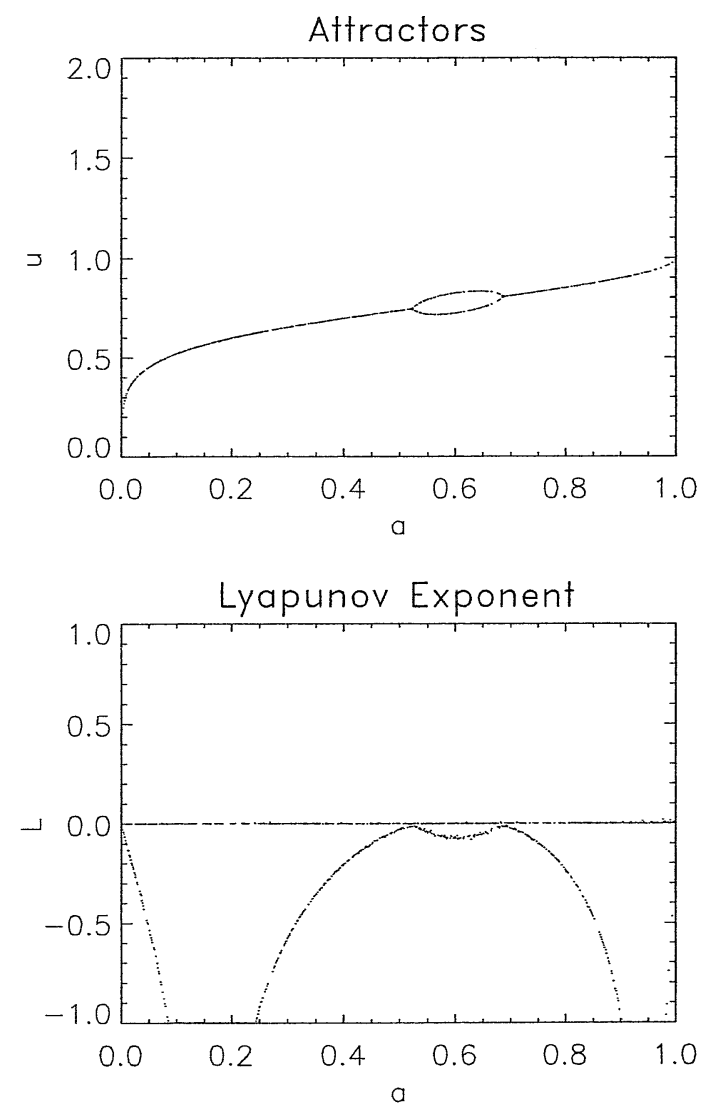

Fig. 2: Attractors and Lyapunov exponent of the radiative map eq. (13) for $\tau=0.15$ and parameters P1 (15).

\subsection{Radiative map}

Considering now the $\tau \neq 0$-case, eq. (7) can be integrated piecewise from one injection time to the next one (using the notation of (8) with $\Delta t \equiv \tau$ ):

$$
u_{i+1}=e^{-\beta \tau} u_{i}+\tau\left[f_{s} \frac{1-e^{-\beta \tau}}{\beta \tau}-e^{-\beta \tau} f_{q} q\left(u_{i}\right)\right] \quad(\tau \text { arbitrary })
$$




$$
\stackrel{\beta \rightarrow 0}{\longrightarrow} u_{i}+\tau\left[f_{s}-f_{q} q\left(u_{i}\right)\right]
$$

This radiative map (13) is valid for arbitrary $\tau$ and identical with (9) for $\Delta t \equiv \tau \rightarrow 0 .^{2}$ In what follows we assume $\beta=0$.
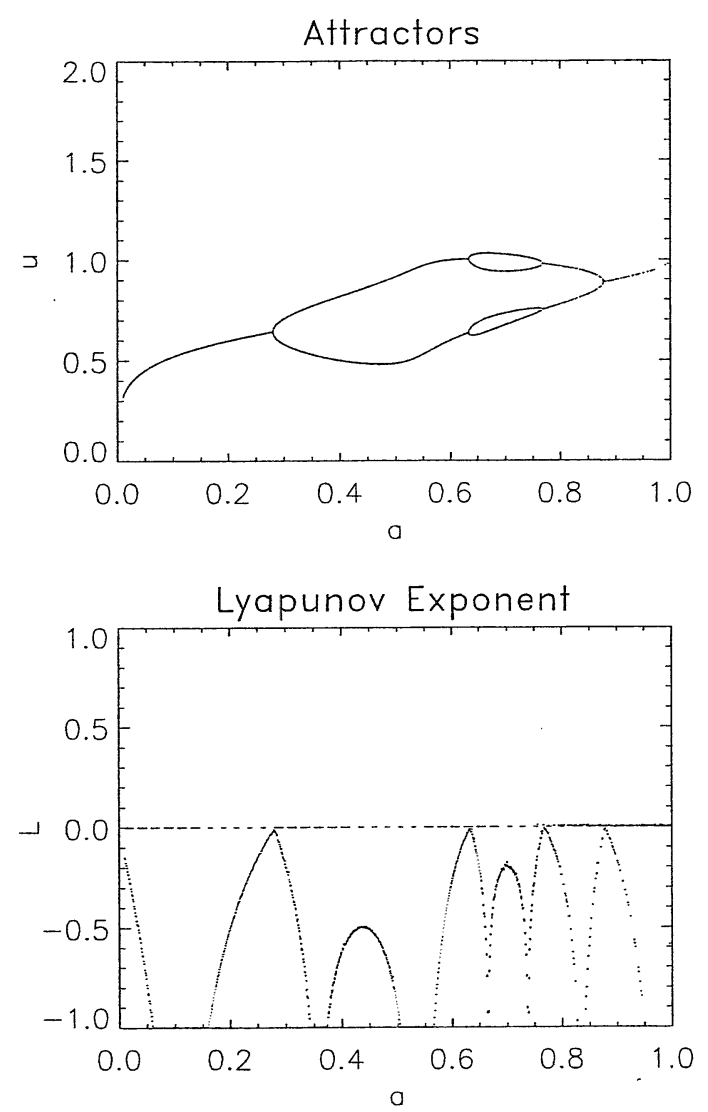

Fig. 3: Attractors and Lyapunov exponent of the radiative map eq. (13) for $\tau=0.2$ and parameters P1 (15).
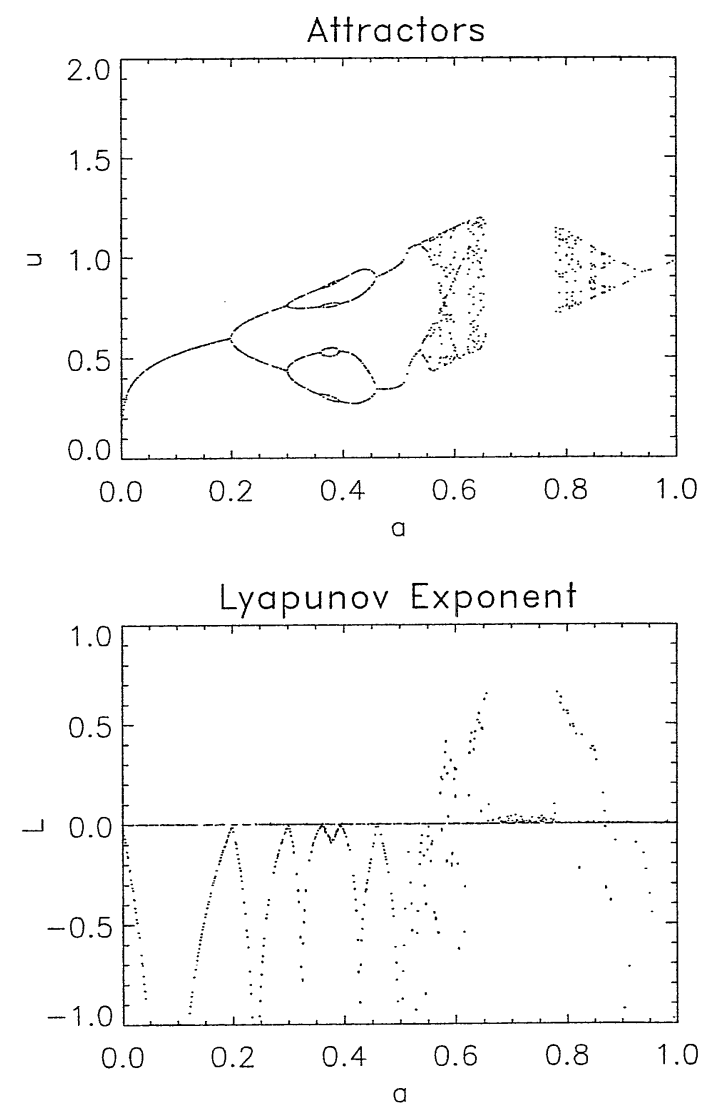

Fig. 4: Attractors and Lyapunov exponent of the radiative map eq. (13) for $\tau=0.25$ and parameters P1 (15).

\subsection{Attractors, period doubling and chaos}

Here we outline the transition to chaos via period doubling phenomena. At first the simple problem characterized by the following parameter set P1 will be solved:

$$
\kappa=\frac{1}{2}(\delta=0), M=1, c_{1}=1, u_{1}^{*}=1, \Delta_{q 1}=0.1, f_{q}=5, f_{s} \equiv 5 a ; a=0 . .1,
$$

where $a$ acts as control parameter. I.e. we consider, for simplicity, the case of constant heating and a radiation loss by a single-humped radiation function with homogeneous impurity density. In Fig. 1 we show the roots of eq. (11) together with the

\footnotetext{
${ }^{2}$ There are some analogies of the well-known circle map, standard and dissipative standard map ("periodically kicked rotator") (e.g. [14]) (this was the reason to introduce the damping constant $\beta$ ) with the radiative map (13) such that the latter can be denoted as "periodically kicked radiator".
} 
attractors of the radiative map (13) for $\tau=0.1$. As can be seen, the upper branch of the roots $u_{1}^{0}(11)$ is unstable according to (12) $\left(L_{1}>0\right)$. Attractors exist only for $a<1$.

The attractor and Lyapunov exponent calculations in Fig. 2 for P1 and $\tau=0.15$ show stable periodic solutions in the vicinity of $a=0.6$. Enhancing $\tau$ further to $\tau=0.2$ shows bubbles, Fig. 3. To be seen are bifurcation points, Lyapunov exponent equal 0 , and super stable cycles where the Lyapunov exponent tends to $-\infty$.

Enhancing $\tau$ further to 0.25 (Fig. 4) leads to chaos with open windows. The dependence of the attractors and Lyapunov exponent on $\tau$ for P1 with $a=0.4$ are depicted in Fig. 4. This is the well-known Feigenbaum route with increasing $\tau$.

The foregoing results are summarized by representing Lyapunov exponent contours in Fig. 6 depending on $a$ (abscissa) and $\tau$ (ordinate). The different regimes of super stable, stable, quasiperiodic and chaotic regions can be seen; also stable oscillations in chaotic regimes (windows) exist.
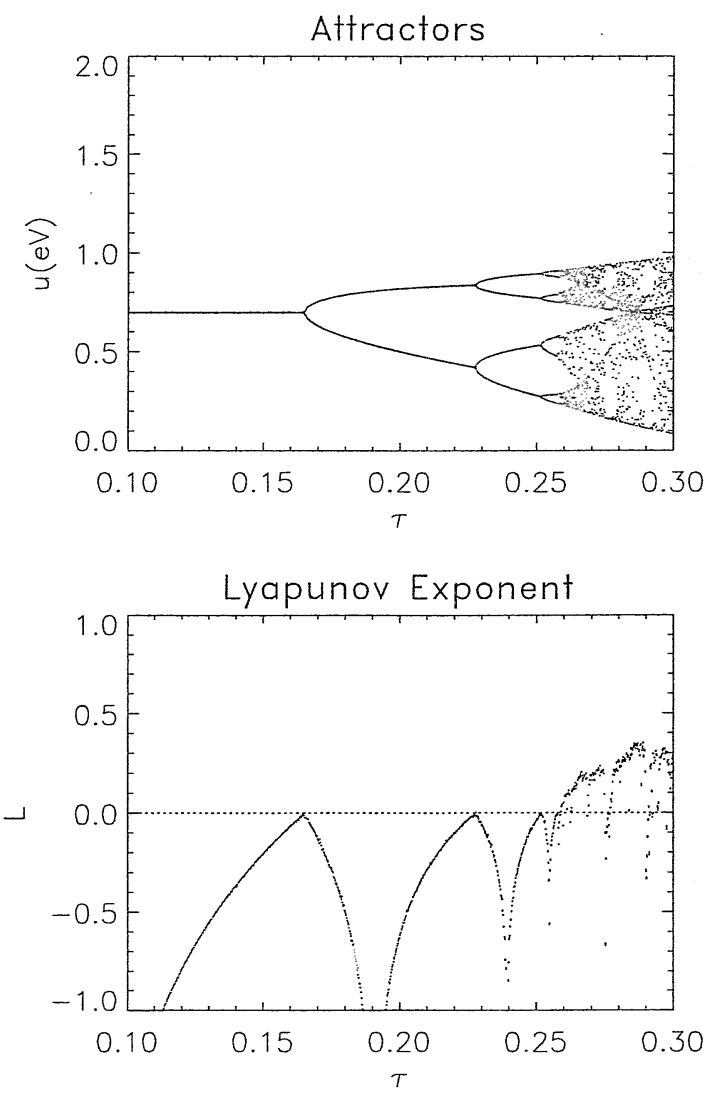

Fig. 5: Attractors and Lyapunov exponent of the radiative map eq. (13) in dependence of $\tau$ for parameters P1 (15) with $a=0.4$. 


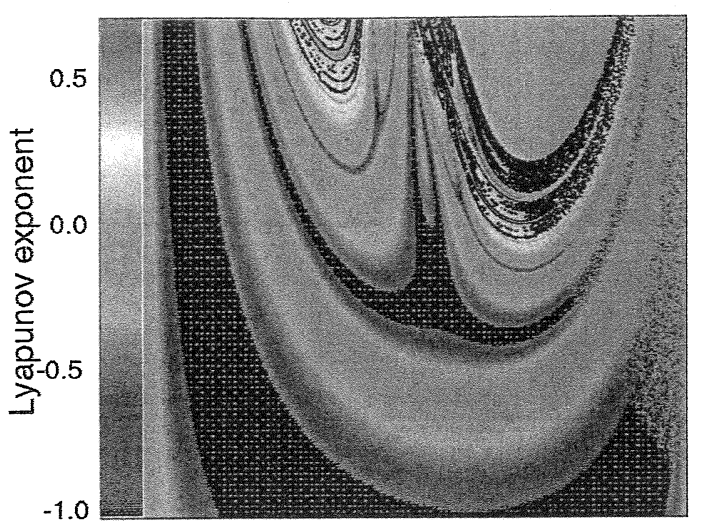

Fig. 6: Lyapunov exponent

Contours of the Lyapunov exponent of the radiative map eq. (13) in dependence of $a=0-1$ (abscissa) and $\tau=0.1-0.3$ (ordinate) for parameters P1 (15).
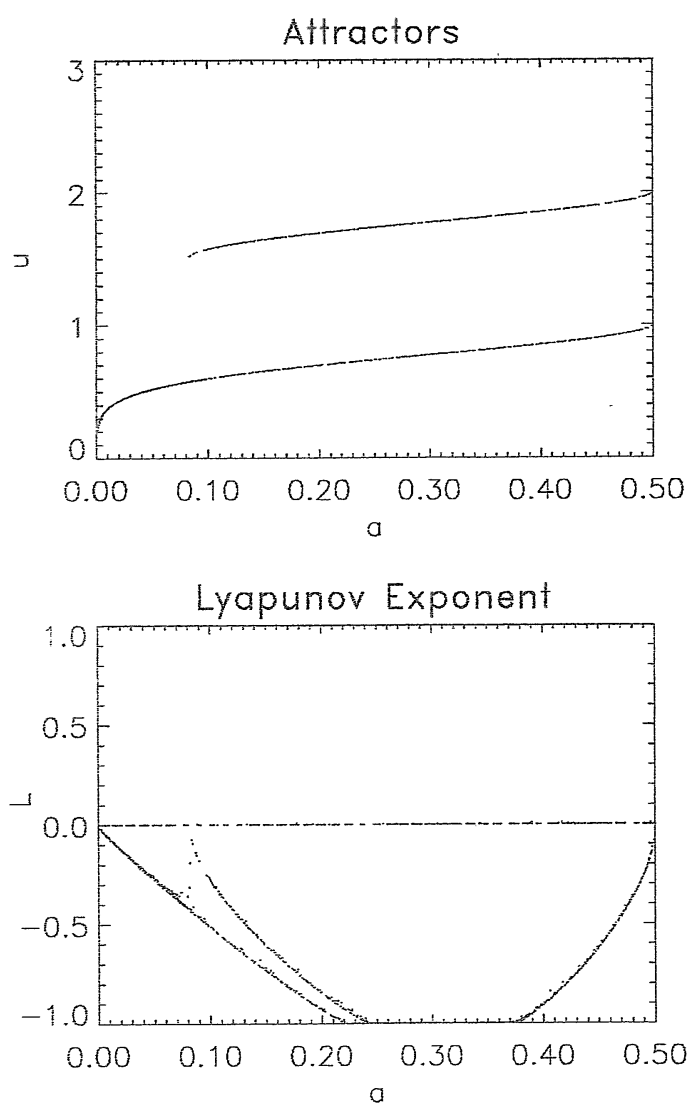

Fig. 8: Attractors and Lyapunov exponent of the radiative map eq. (13) for $\tau=0.1$ and parameters P2 (16).

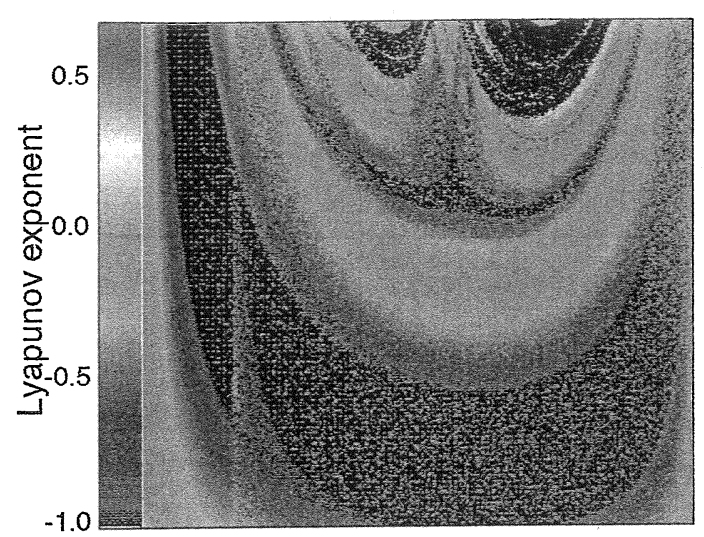

Fig. 7: Lyapunov exponent

Contours of the Lyapunov exponent of the radiative map eq. (13) in dependence of $a=0-0.5$ (abscissa) and $\tau=0.1-0.5$ (ordinate) for parameters P2 (15).
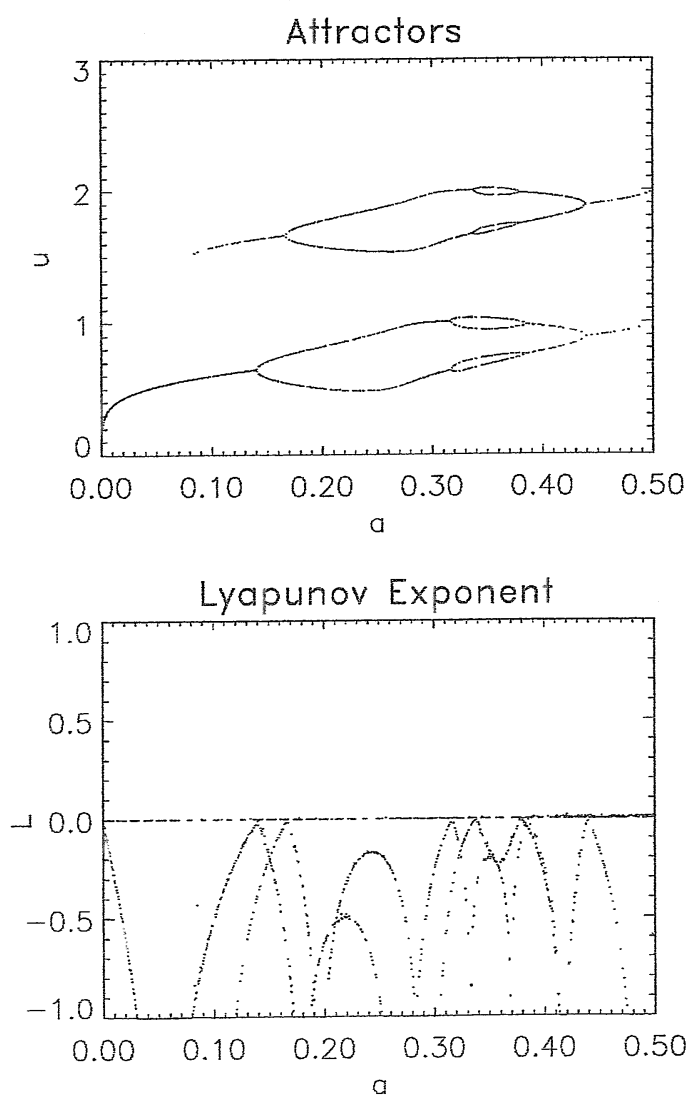

Fig. 9: Attractors and Lyapunov exponent of the radiative map eq. (13) for $\tau=0.4$ and parameters P2 (16). 
We turn now to the case of a radiation function with two maxima, $M=2$. This can be realized e.g. by two impurities or a double-humped radiation function for e.g. carbon (sect. 6). Here we consider a double-humped radiation function with equal fractions, $c_{1,2}=0.5$, parameters $\mathbf{P} 2$ :

$$
\begin{array}{r}
\kappa=\frac{1}{2}(\delta=0), M=2, c_{1}=0.5, u_{1}^{*}=1, \Delta_{q 1}=0.1, f_{q}=5, \\
c_{1}=0.5, u_{2}^{*}=2, \Delta_{q 1}=0.1, f_{s} \equiv 5 a ; a=0 . .1
\end{array}
$$

There is one attractor in the range $0<a \lesssim 0.1$, and two for $0.1 \lesssim a<0.5$ (cp. Fig. 8). Attractors and Lyapunov exponent for P2 and $\tau=0.1$ are shown in
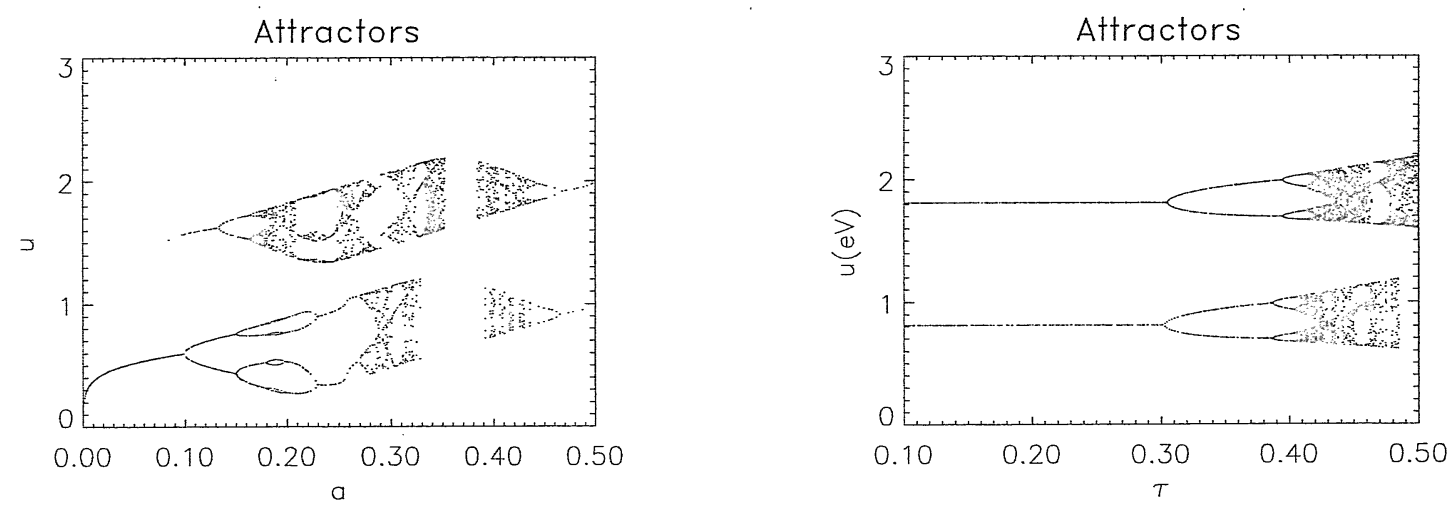

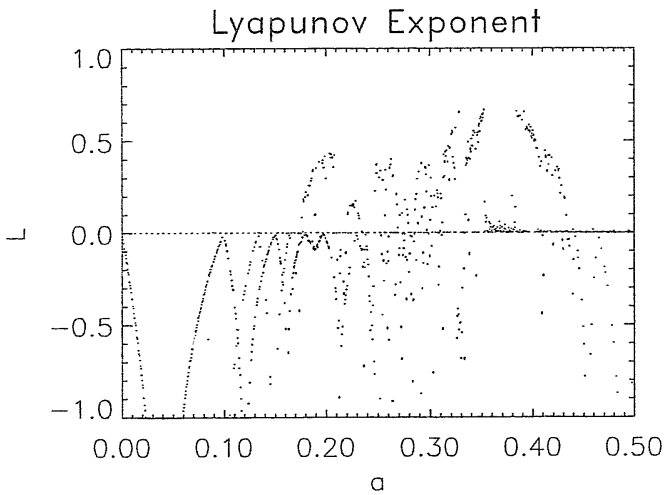

Fig. 10: Attractors and Lyapunov exponent of the radiative map eq. (13) for $\tau=0.5$ and parameters P2 (16).

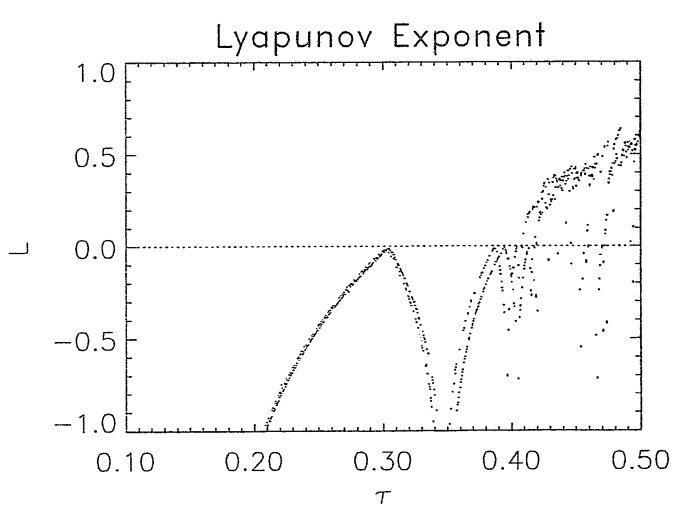

Fig. 11: Attractors and Lyapunov exponent of the radiative map eq. (13) in dependence of $\tau$ for parameters P2 (15) with $a=0.35$.

Fig. 8. The two attractor branches correspond to the two maxima of the radiation function. Their strengths are determined by the relevant fraction $c_{i}$. For P2 those ones are approximately equal. Analogous to the $M=1$ case, Fig. 1 , there exist two repellors, where the lower one connects the two attractors. Perturbing this repellor e.g. periodically may lead to relaxations between the two attractors. Fig. 9 shows bubbles for P2 and $\tau=0.4$. Chaotic results can be seen in Fig. 10 (P2 and $\tau=0.5$ ). There are some open windows which allows a variety of stable periodic solutions. In Fig. 11 the double Feigenbaum route with increasing $\tau$ can be seen for $a=0.35$. 
In Fig. 7 the Lyapunov exponent contours are displayed in dependence of a (abscissa) and $\tau$ (ordinate). Compared to Fig. 6 the contours show an analogous behaviour but are multi-layered due to the two maxima of the radiation function.

\section{Steady problem}

The reason to discuss the steady problem $(\tau=0)^{3}$ is that we are concerned with the solution of spatial boundary value problems and their temporal evolution. The steady heat conduction equation follows from eq. (1) for $\partial / \partial t=0$ :

$$
\frac{d}{d x} \kappa(u) \frac{d u}{d x}+f(u, x)=0, x \in\left[x_{0}, x_{n}\right] \quad\left(x_{0}=0, x_{n}=1\right), \quad \Theta_{\tau}=1
$$

The following mixed boundary conditions are imposed:

$$
\left[\kappa(u) \frac{d u}{d x} \mp \alpha_{0, n} u^{\beta_{0, n}}\right]_{x=x_{0, n}}=0
$$

where Dirichlet's and Neumann's conditions are contained as special cases.

We investigate bifurcation phenomena by using phase plane analysis, i.e. we try to find solutions of $(17)$ in the $\left(u, u_{x}\right)$-plane where $x$ is the curve parameter.

The topology of the phase space portraits is determined by the parameters of eq. (17), and is dependent on the fact whether or not this equation has singular points. These points can also be calculated analytically for the simple case considered above for the temporal problem, eqs. (11), $(12)^{4}$ :

$\underline{f_{s}>f_{q}}-$ no rest points

$\frac{f_{s}<f_{q}}{L^{(1,2)}}$ - two rest points $P_{1,2}\left(P \equiv\left(u, u_{x}\right)\right.$ - phase plane point) with the eigenvalues $P_{1}$ (saddle):

$$
u_{1}^{0}=u_{1}^{*}-\sqrt{\Delta_{q 1} \ln \frac{f_{q}}{f_{s}}}, u_{x 1}^{0}=0, \quad L_{1,2}^{(1)}= \pm \sqrt{\frac{1}{\kappa_{0}} \frac{2 f_{s}}{\sqrt{\Delta_{q 1}}} \ln \frac{f_{q}}{f_{s}}}
$$

$P_{2}$ (vortex):

$$
u_{2}^{0}=u_{1}^{*}+\sqrt{\Delta_{q 1} \ln \frac{f_{q}}{f_{s}}}, u_{x 2}=0, \quad L_{1,2}^{(1)}= \pm i \sqrt{\frac{1}{\kappa_{0}} \frac{2 f_{s}}{\sqrt{\Delta_{q 1}}} \ln \frac{f_{q}}{f_{s}}}
$$

Thus, for $f_{s}<f_{q}$ we have an island structure, for $f_{s}>f_{q}$ we have none. Hence, the equation $f_{s}=q_{\max }=f_{q}$ defines a bifurcation point in the control space. This result can easily be generalized for the case of several impurities $M>1$ where chains of $M$ saddles/vortices may appear. Different from the temporal case (12), here the eigenvalues are also determined by the heat conduction coefficient $\kappa_{0}$.

\footnotetext{
${ }^{3} \mathrm{Cp} .[7]$.

${ }^{4}$ The temporal problem is of first-order, the spatial of second-order.
} 
We solve the boundary value problem

$$
\alpha_{0, n}=1.6, \beta_{0, n}=0.5
$$

for P1 (15) with $a=0.2 \ldots 1.2$.
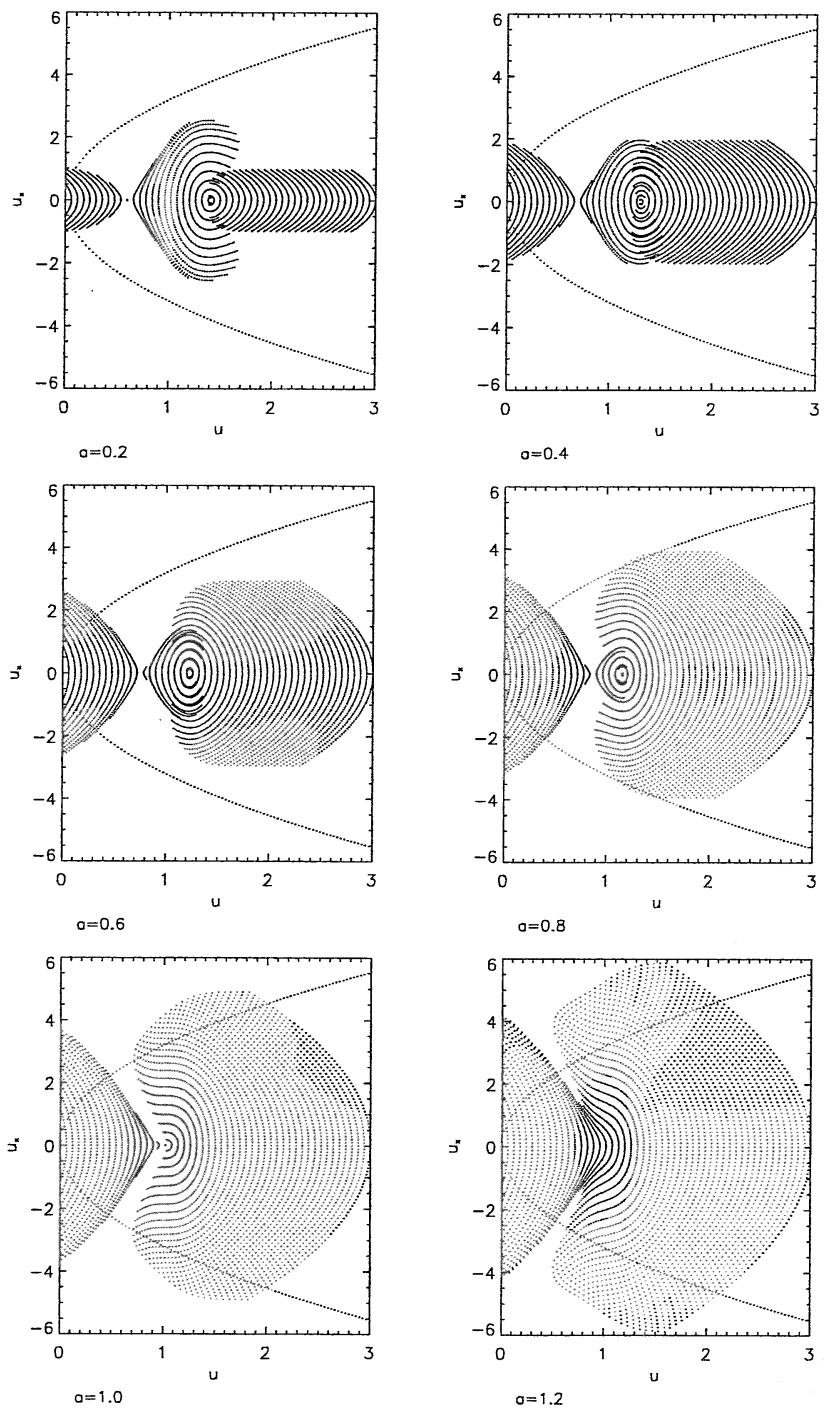

Fig. 12: Phase plane portraits $\left(u, u_{x}\right)$ for parameters P1 (15) with $a=0.2 \ldots 1.2$ as control parameter together with the boundary value curves according to (18), (21).

Fig. 12 shows phase plane portraits $\left(u, u_{x}\right)$ for parameters P1 (15) with $a=0.2 \ldots 1.2$ as control parameter together with the boundary value curves according to (18), (21); the curve parameter is $x \in[-0.5,0.5]$. Solutions of the considered boundary value problem are only those trajectories which start and end on the respective boundary value curve. As can be seen there is only one solution for $a=0.2 \ldots 0.6$ and three solutions for $a=0.8 \ldots 1.2$. The solution of boundary value problems can lead to a non-trivial phase plane structure resulting in rather complicated solution manifolds which shall undergo 'chaotic impacts'. 


\section{Spatio-temporal chaos}

Having discussed Feigenbaum routes to chaos by considering the temporal problem in sect. 3, and shortly bifurcation properties of steady systems with respect to boundary value problems, let us now turn to chaotic phenomena in $1 \mathrm{~d}$ plasmas, i.e. look for solutions of the fully time dependent $1 \mathrm{~d}$ heat conduction equation (1) on the $x$ domain $[0,1]$. We consider the time evolution of temperature profiles due to a localized heat source and radiation loss by an $M=1$ radiation loss function, and apply a simple Dirichlet boundary condition which is not so sensitive to imposed changes $\left(\alpha_{0, n}=\right.$ $\left.0, \beta_{0, n}>0\right)$. The following parameter set $\mathrm{P} 3^{5}$ is used:

$$
\kappa=\frac{1}{2}, M=1, c_{1}=1, u_{1}^{*}=1, \Delta_{q 1}=0.1, f_{q}=7, x^{*}=0.5, \Delta_{s}=0.05, f_{s}=15
$$
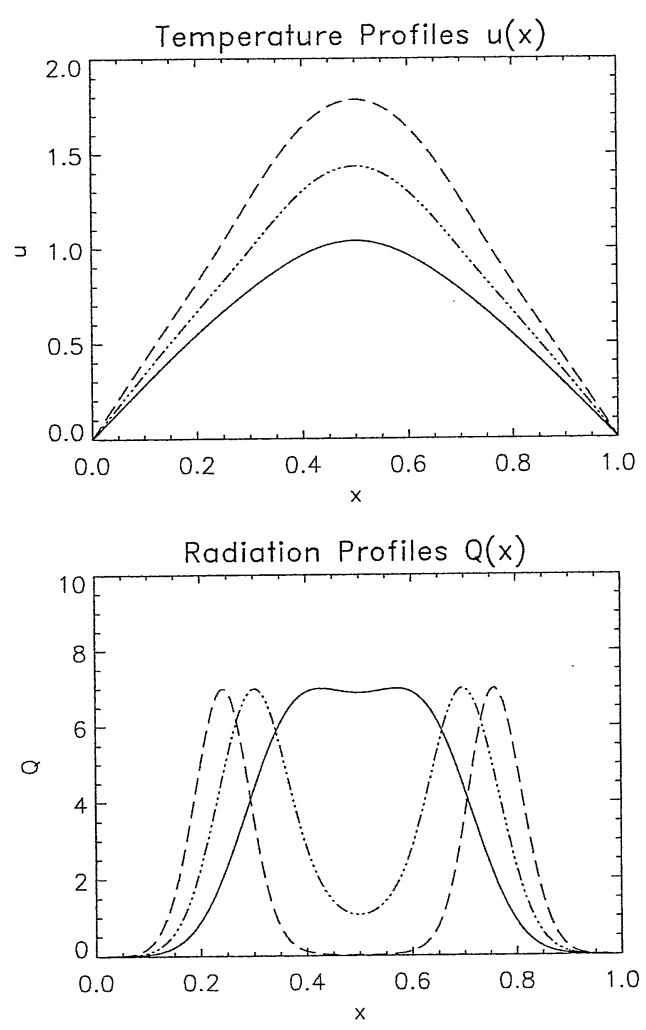

Fig. 13: Steady temperature profiles (attractors: dashed and full line, repellor: dotted dashed line) and corresponding radiation profiles for parameters P3 (22).

At first the steady problem P3 with $\Theta_{\tau}=$ 1 is considered. As it can be understood by the explanations of the foregoing section, the temperature profile calculations in Fig. 13 show that there are three solutions for the boundary value problem where the middle (unstable) solution acts as repellor, the two others are attractors. Thus, dependent on the initial profile one of the two attractors will be attained. The corresponding three radiation profiles are also displayed.

In what follows we solve the spatiotemporal problem P3 for different initial profiles and increasing $\tau$. We compute spatially extended attractors, and the time evolution in the centre $(x=0.5)$ of the $x$ domain. For the present the radiation loss function is assumed to be nonlocalized $\left(c_{1}=\right.$ const in P3 (22)).

In Figs. 14, 15 attractors (the dotted lines are the initial profiles) and the temperature evolution at $x=0.5$ are displayed starting at different initial profiles. As compared with the steady solutions, Fig. 13, each attractor splits into two, i.e. the (local) fix point changes via limit cycles (Hopf bifurcation) to oscillations which show intermittency.

Enhancing $\tau$ further the two starting attractors can not be separated. In Fig. 16 temperature attractors are shown for P3 (22) and $\tau=0.1$. They have a weakly spatial structure. Also shown are the corresponding radiation envelopes. The temperature evolution together with a phase space representation are shown in Fig. 17 for the

\footnotetext{
${ }^{5}$ See the discussion of bifurcation and hysteresis effects in [3]
} 
same parameters. To get this simple overview the time delayed method is used by representing the phase plane trajectories $[u(t), u(t+\tau / 4)]$. The time-chaotic behaviour is obvious.

Clearly, enhancing $\tau$ further leads to unsolvable boundary value problems.

Up to now we treated only spatially homogeneous radiation sinks. At last we consider impurities localized near the edge of the $x$ domain at $x=0.2\left(c_{1}=2.5\right.$ for $1.5 \leq x \leq 2.5$ and 0 otherwise). It results a radiation peak at $x=0.2$ with attractors and oscillations there that can be seen in Fig. 18.
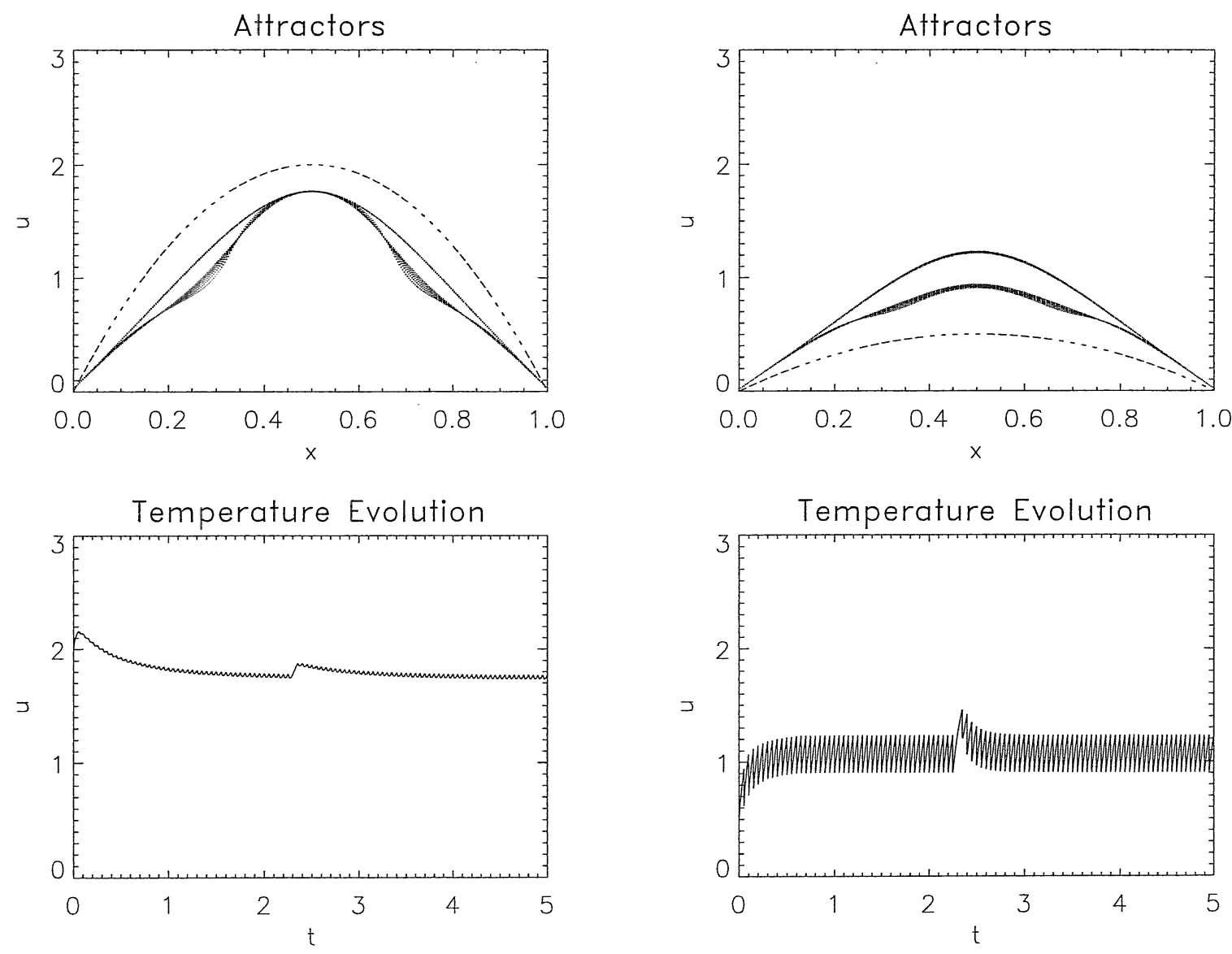

Fig. 14: Attractors for $\tau=0.05$ in dependence of $x$ (the dotted line - initial profile) and temperature $u$ as a function of $t$ at $x=0.5$ for P3 (22).

Fig. 15: Attractors for $\tau=0.05$ in dependence of $x$ (the dotted line - initial profile) and temperature $u$ as a function of $t$ at $x=0.5$ for P3 (22). 

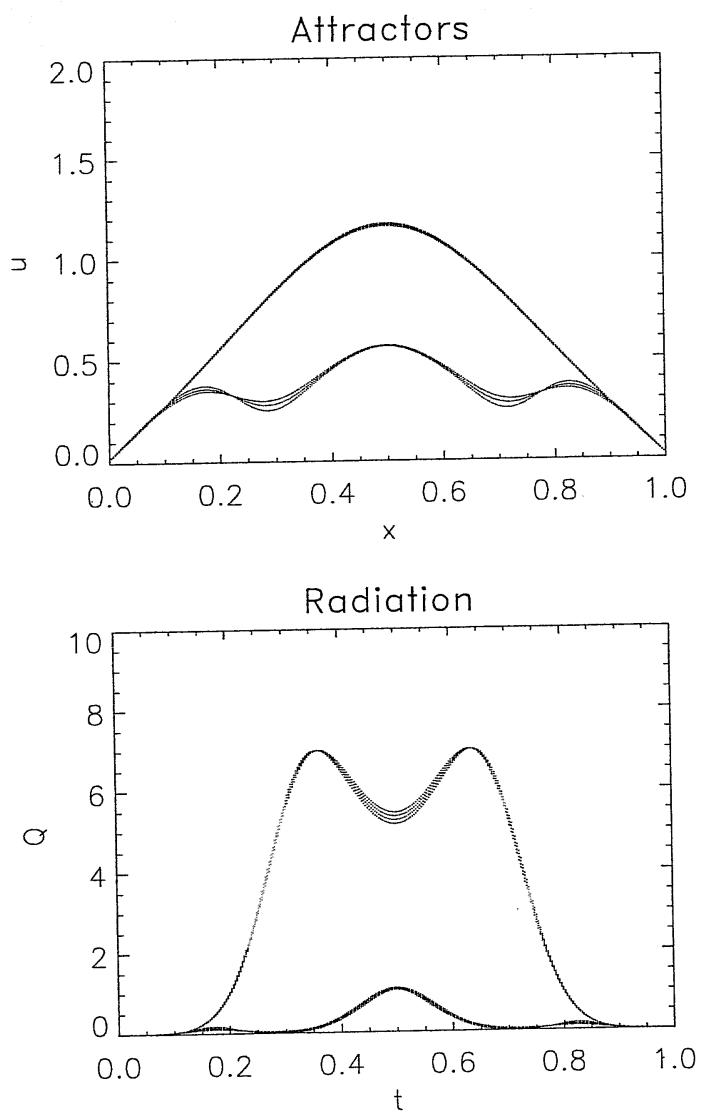

Fig. 16: Attractors for $\tau=0.1$ in dependence of $x$ and corresponding radiation function for P3 (22) and $\tau=0.1$.

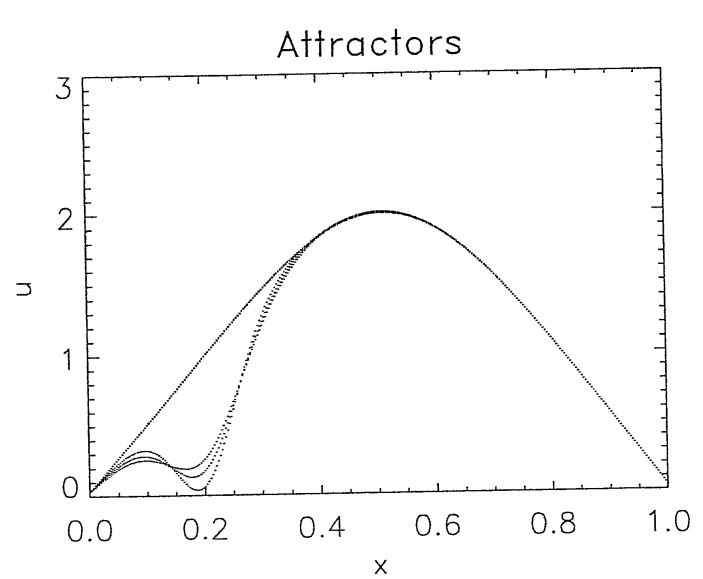

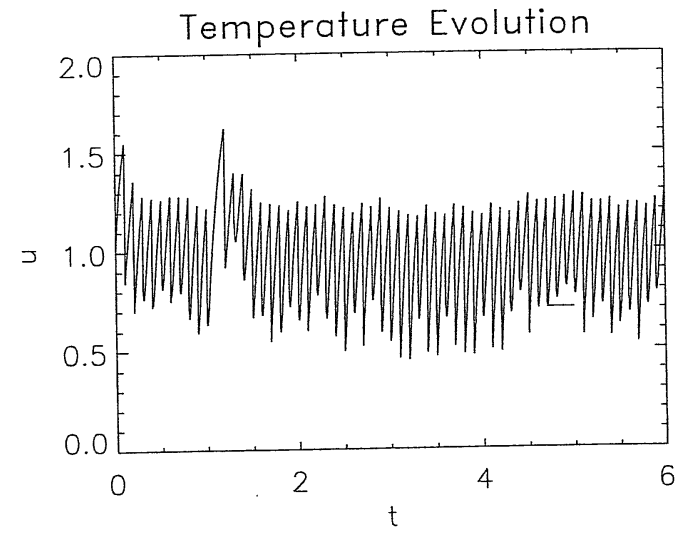

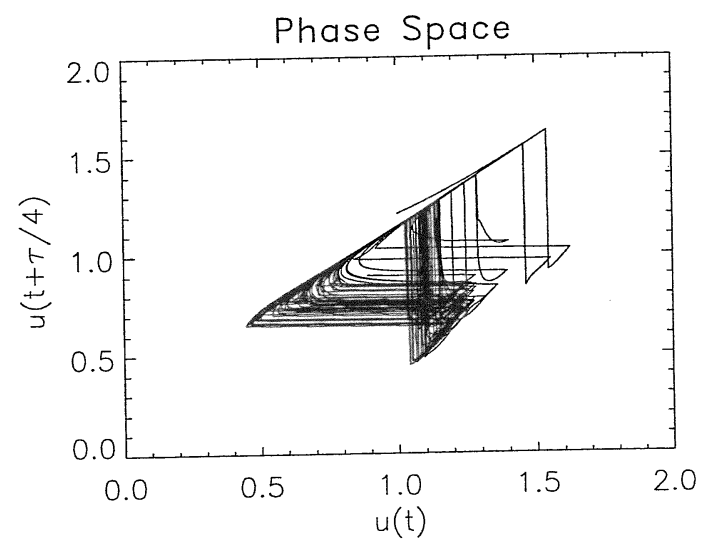

Fig. 17: Temperature $u$ as a function of $t$ and phase space representation at $x=0.5$ for P3 (22) and $\tau=0.1$.

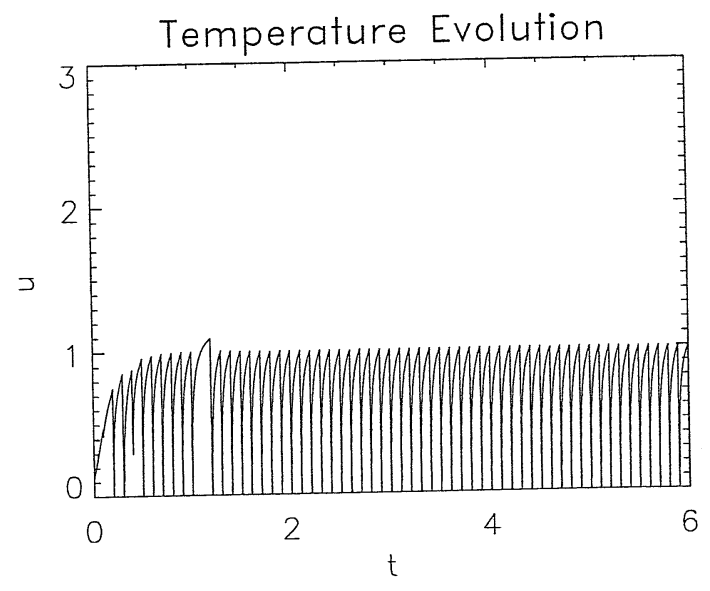

Fig. 18: Attractors for $\tau=0.1$ in dependence of $x$ and temperature $u$ as a function of $t$ for P3 (22) at $x=0.2$. 


\section{Application to Carbon}

Let us now applicate our results to a carbon seeded plasma with parameters of the divertor plasma of the ASDEX Upgrade tokamak, cp. [9]: For a power input $P_{d i v}=0.95 \cdot 10^{6} \mathrm{~W}$ into the divertor region of a volume $V_{d i v}=6 \cdot 10^{5} \mathrm{~cm}^{3}$ we have $\beta \equiv P_{\text {div }} / V_{\text {div }}=1.583 \cdot \mathrm{W} / \mathrm{cm}^{3}$, resulting in $f_{s}=\frac{\beta}{3 n_{e}}$ with the electron density given by $n_{e}=n_{i}+Z n_{z}, n_{i}$ - ion density. All the densities are now parameters. The radiation loss term is given according to eq. (5) with the radiation function $Q$ given by the ADPAK data [15] (neglecting the effect of the neutrals).

Parameters P4:

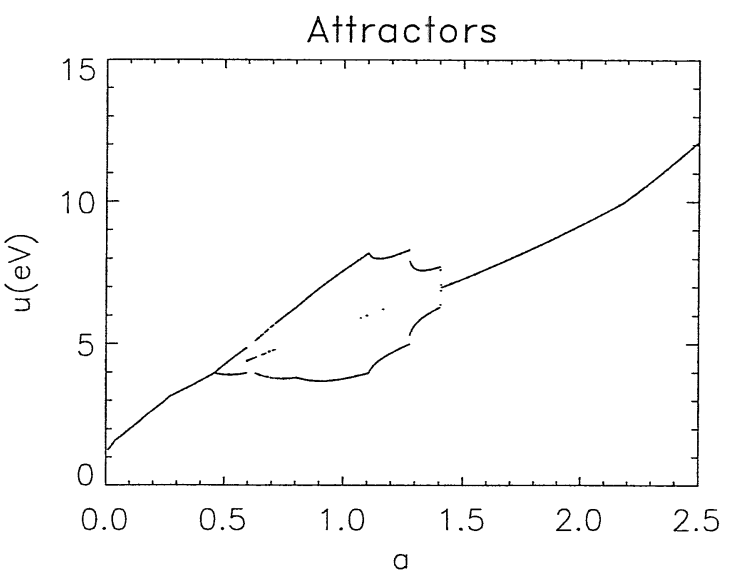

Fig. 19: Attractors of the radiative map eq. (13) for Carbon with parameters P4 (23).

$$
n_{i}=0.5 \cdot 10^{14} \mathrm{~cm}^{-3}, n_{z}=0.5 \cdot 10^{12}, \tau=10^{-4} s, f_{s}=\frac{\beta}{3 n_{e}} a, a=0-2.5
$$

$u$ now is the temperature in $\mathrm{eV}, \tau$ the time period in $\mathrm{s}$.

Here $n_{z}<<n_{e}$ (clean plasma). Computing the attractors for P4, Fig. 19 shows the typical behaviour in dependence of the parameter $a$ as seen above: There are only attractors in the low temperature regime $\lesssim 10 \mathrm{eV}$ for $a=0-2.5$. For $a$ in the vicinity of 1 we obtain the regime of small relaxation oscillations [9].

Parameters P5 (dirty plasma):

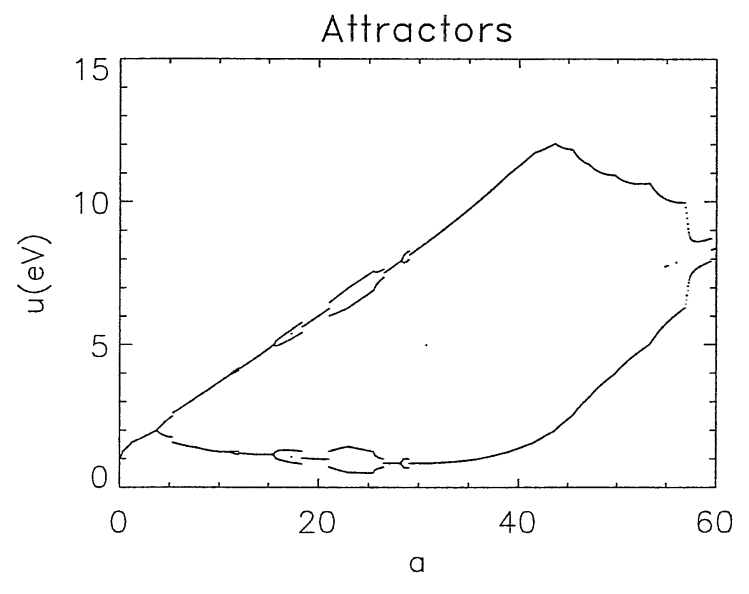

Fig. 20: Attractors of the radiative map eq.

(13) for Carbon with parameters P5 (24).

$$
n_{i}=0.5 \cdot 10^{14} \mathrm{~cm}^{-3}, n_{z}=0.96 \cdot 10^{13}, \tau=6 \cdot 10^{-6} s, f_{s}=\frac{\beta}{3 n_{e}} a, a=0-50
$$

The attractors to P5 are shown in Fig. 20. They are rather chaotic with some bubbles. There are a variety of oscillation regimes. For $\tau<3 \mu$ s there are no oscillations.

Of course, the connection to the relaxation phenomena of [9] is important ${ }^{6}$, also a spatio-temporal description analogous to sect. 5 .

\footnotetext{
${ }^{6}$ This will be discussed in a forthcoming paper [12].
} 


\section{Summary}

Bifurcation phenomena of the temperature are of great importance in radiative edge plasmas of tokamaks and stellarators. In the frame of a bifurcation treatment low-frequency relaxation oscillations observed in divertor plasmas of the ASDEX Upgrade tokamak [8] have been interpreted in [9] by the use of a simple 0d model. In order to investigate radiative properties of edge plasmas we make use of the model [9]. But we assume a relaxation-like temporal modulation of the impurity density with the time period $\tau$ given and investigate its effect on the temperature governed by the heat conduction equation only. The density modulation is described in the simple form of periodic delta function behaviour. This gives us the opportunity to take an arbitrary modulation of the impurity density into consideration that can drive a bifurcation leading to chaotic temperature behaviour.

Before investigating the routes to chaos in the spatio-temporal system we consider at first the time dependent problem. Our ansatz allows to derive a simple radiative map which shows the routes to chaos via period doubling phenomena. Attractors and Lyapunov exponents are calculated for a variety of parameter sets. The main parameters varied are the power input and the time period $\tau$. It was shown that increasing both parameters forces chaos.

By solving the time dependent $1 d$ heat conduction equation we are concerned with spatial boundary value problems that give rise to bifurcation phenomena and multiple solutions, sect. 4. We consider a simple Dirichlet problem having two attractors and one repellor as steady solution profiles $(\tau \rightarrow 0)$. The effect of density modulation by increasing $\tau$ is an attractor splitting which leads via Hopf bifurcation and intermittency to temporal chaos, and at last to unsolvable boundary value problems. The attractors have also a weakly spatial structure. An example of a spatially localized density profile is also given.

Our results are applicated to a carbon seeded plasma with parameters of the divertor plasma of the ASDEX Upgrade tokamak, cp. [9]. The mentioned low-frequency relaxation oscillations are found in a clean operating regime. Enhancing the impurity density (dirty regime) forces chaos. An 1d treatment remains. 


\section{References}

[1] Capes, H., Ghendrih, Ph., Samain, A., Phys. Fluids B4 (1992) 1287

[2] D. Sünder, H. Wobig, 20th EPS, Lisboa 1993, Contrib. Papers II-819

[3] P. Bachmann, D. Sünder, H. Wobig, 24th EPS, Berchtesgaden 1997, Contrib. Papers IV -1817

[4] P. Bachmann, D. Sünder, H. Wobig, Contrib. Plasma Phys. 38 (1998) 1/2, 379

[5] D. Sünder, H. Wobig, Bifurcation of Temperature in 3-D Plasma Equilibria, IPP Report 2/243, Nov. 1998

[6] P. Bachmann, D. Sünder, H. Wobig, Contrib. Plasma Phys. 40 (2000) 3-4, 399

[7] P. Bachmann, J. Kisslinger, D. Sünder, H. Wobig, Bifurcation of Temperature in the Boundary Region of Advanced Stellarators, Report IPP III/262, May 2000

[8] U. Wenzel, P. Bachmann, A. Carlson, et al, Nucl. Fusion 37(1997)1343

[9] P. Bachmann, D. Sünder, U. Wenzel Contrib. Plasma Phys. 36(1996)4,519

[10] V.A. Abramov, S.G. Bespoludennov, Yu.L. Igitkhanov, V.I. Pistunovich, J. Nucl. Mat. $176 / 177(1990) 922$

[11] H. Kastelewicz, R. Schneider, J. Neuhauser, Plasma Phys. Control Fusion 37(1995)723

[12] P. Bachmann, U. Wenzel (in preparation)

[13] A.J. Lichtenberg, M.A. Liebermann, Regular and Chaotic Dynamics, Springer Verlag, New York, Berlin, etc., 1992

[14] R. Leven, B.-P. Koch, B. Pompe, Chaos in dissipativen Systemen, Akademie-Verlag 1994

[15] D.Post, R. Hulse, D. Stotler, Contrib. Plasma Phys. 30(1994)300 